\title{
Light-mediated Changes in Pigmentation of Pseudomonas aeruginosa Cultures
}

\author{
By C. PROPST* AND L. LUBIN \\ GTE Laboratories, Inc., Waltham Research Center, Waltham, Massachusetts, U.S.A.
}

(Received 25 January 1979)

Cultures of Pseudomonas aeruginosa PAO grown under uninterrupted broad-spectrum light showed different pigmentation from dark-grown cultures. Whereas dark-grown bacteria produced pigments which resulted in blue-purple coloured agar, light-grown organisms produced red coloured plates. Extraction and quantification of pigments showed that both dark- and light-grown cultures produced similar concentrations of pyorubrin (red) and pyoverdin (yellow). In contrast, the concentration of pyocyanin (blue) was substantially reduced under certain lighting conditions. This decrease was dependent on both the light intensity and wavelength and occurred with light in the ultraviolet and violet region of the spectrum. After its release from bacteria, pyocyanin was rapidly and nonreversibly photoinactivated with first-order kinetics to produce colourless photoproduct(s).

\section{INTRODUCTION}

While studying the general effect of visible and near-visible light on bacteria, we observed that cultures of Pseudomonas aeruginosa produced different coloured agar plates when grown under light and dark conditions. We presumed that this reflected changes in the diffusible pigments present. Since light-mediated effects in micro-organisms are of interest (see Zelle \& Hollaender, 1955; Leach, 1971; Jagger, 1972; Taylor \& Koshland, 1975; Thomas, 1977) and since pigment production is a characteristic commonly used for identifying and differentiating pseudomonads, the following study was undertaken.

\section{METHODS}

Cultures. Pseudomonas aeruginosa strain PAO, obtained from P. V. Phibbs (Virginia Commonwealth University), was used for all studies presented in this paper. Major findings were confirmed using $P$. aeruginosa strain SC8822 obtained from R. J. McRipley (E. R. Squibb).

Growth conditions. Medium A (King et al., 1954) was used in this study. This medium was supplemented with $1 \mathrm{~mm}-\mathrm{CuCl}$ to enhance pigment production (King et al., 1954). Similar results were obtained with Pseudosel agar (BBL), except that production of pyorubrin was depressed. Disposable plastic Petri dishes (approx. $10 \times 50 \mathrm{~mm}$; Millipore) containing $4 \mathrm{ml}$ medium were surface inoculated with about $2 \times 10^{7}$ bacteria. Cultures were grown at $25^{\circ} \mathrm{C}$ for specified periods in the presence or absence of light (see below). The plastic Petri dishes transmitted $90 \%$ of the incident light above $360 \mathrm{~nm}$.

Assays. Pigments were extracted and assayed as previously described by Palumbo (1972). Basically, the technique involved chloroform extraction of the medium to separate pyocyanin, followed by aqueous extraction of the medium to remove pyoverdin and pyorubrin together. For spectrophotometric determination, pyocyanin was extracted from chloroform into $0.2 \mathrm{M}$-aqueous $\mathrm{HCl}$, and absorbance at $660 \mathrm{~nm}$ was determined after neutralization to $\mathrm{pH} 7 \cdot 0$. Pyorubrin was measured in aqueous extracts by absorbance at $525 \mathrm{~nm}$. Pyoverdin was measured in aqueous extracts by fluorescence at $525 \mathrm{~nm}$ after excitation at $410 \mathrm{~nm}$ (pH $8 \cdot 0$ to $8 \cdot 2$ ).

* Present address: Abbott Laboratories, Biological Development, Dept 456/Bldg R1b, 14th St and Sheridan Rd, North Chicago, Illinois 60064, U.S.A. 


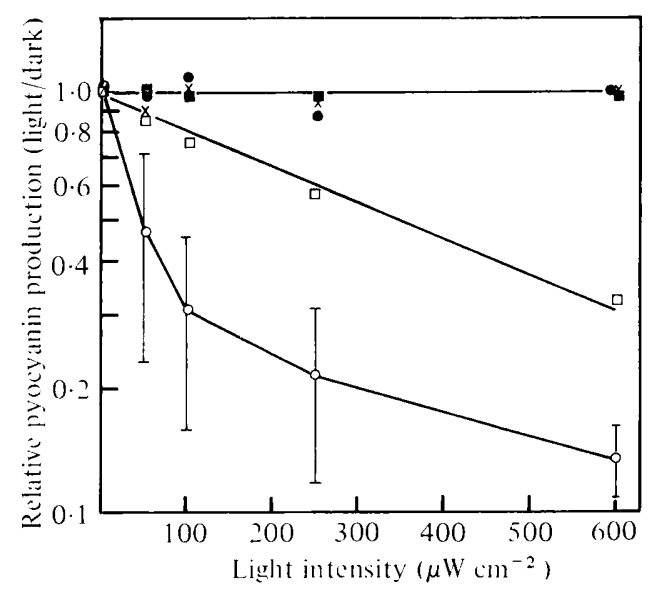

Fig. 1. Effect of light wavelength and intensity on pyocyanin production. Bacteria were inoculated on to plates and grown under light of different wavelengths $(\bigcirc, 371 \mathrm{~nm} ; \square, 420 \mathrm{~nm} ; \times, 550 \mathrm{~nm}$; $\square, 660 \mathrm{~nm} ; 0,740 \mathrm{~nm}$ ) at the specified intensities for $5 \mathrm{~d}$. Results are expressed as the ratio of pigment produced under lighted conditions to pigment produced by dark-grown controls. Representative ranges $( \pm$ one standard error) for each point have been calculated for $371 \mathrm{~nm}$ light. Results are averages of three experiments.

Lighting. One broad-spectrum (350 to $700 \mathrm{~nm})$ and five narrow-bandwidth $(371,420,550,660$ and $740 \mathrm{~nm})$ fluorescent lights were used. Light intensity was controlled by adjusting the distance between the lights and the irradiated object. Irradiance was measured at the level of the object using a Tektronix J16 Photometer/ Radiometer with a J6512 probe. The spectral characteristics of these lights and the techniques for measurement of light intensity have previously been described in detail (Propst-Ricciuti \& Kenny, 1974; Siebert et al., 1975).

\section{RESULTS}

General response of $P$. aeruginosa to broad-spectrum light

Pseudomonas aeruginosa PAO grown on agar medium in the dark normally produces three characteristic diffusible pigments: (1) pyocyanin, a non-fluorescent blue pigment; (2) pyorubrin, a non-fluorescent red pigment; (3) pyoverdin (fluorescin), a fluorescent yellow pigment. Studies of the effects of broad-spectrum light $\left.(2400 \mu \mathrm{W} \mathrm{cm})^{-2}\right)$ on $P$. aeruginosa grown for $4 \mathrm{~d}$ at room temperature showed dramatic changes in pigmentation. Whereas dark-grown organisms produced diffusible pigments which resulted in blue-purple coloured agar, light-grown organisms produced red coloured plates. The pigments produced were extracted and quantified. The ratios ( \pm one standard error) of the amounts of pyocyanin, pyorubrin and pyoverdin produced by light-grown organisms to the amounts produced by dark-grown organisms were, respectively, $0.52 \pm 0.02,0.99 \pm 0.02$, and $0.94 \pm 0.05$. Thus only pyocyanin was affected by broad-spectrum light under the conditions tested.

\section{Effect of light wavelength and intensity on pigmentation}

To determine the effects of the wavelength and intensity of light on the decrease in pyocyanin concentration and to investigate more fully the possible effect of light on pyorubrin and pyoverdin, cultures of $P$. aeruginosa were grown under different intensities of light at $371,420,550,660$ and $740 \mathrm{~nm}$ for $5 \mathrm{~d}$ and then pigments were extracted and quantified. In support of the results obtained with broad-spectrum light, pyorubrin and pyoverdin were either unaffected or only marginally influenced by light intensity and wavelength when studied from 0 to $600 \mu \mathrm{W} \mathrm{cm}-2$ with the above narrow-bandwidth fluorescent lights (data not presented). The concentration of pyocyanin, however, was substantially reduced under 


\section{Table 1. Effect of treatment conditions on pyocyanin concentration}

The medium used for plates was not exposed to light after preparation, except as indicated. Pre-treated plates were irradiated for $3 \mathrm{~d}$ just prior to inoculation. After inoculation, all plates were incubated for $3 \mathrm{~d}$ under light or dark conditions, as appropriate. Bacteria were removed from the agar surface of all plates by flushing with distilled water prior to extraction procedures. For post-treated plates, bacteria were removed from the agar surface by first flushing with sterile distilled water, then with $95 \%$ ethanol to prevent regrowth and finally with more sterile distilled water (control studies had shown that this procedure did not affect the coloration of agar plates); the plates were

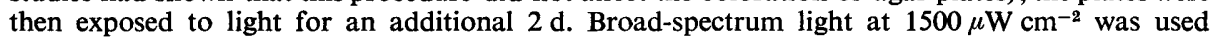
throughout. Results are averages of three experiments \pm one standard error.

$\begin{array}{lllll}\text { Medium treatment/growth conditions } & & \begin{array}{c}\text { Pyocyanin } \\ \left(A_{660} \text { units }\right)\end{array} \\ \text { Pre-treated } & \text { Growth } & \text { Post-treated } & \text { Plate colour } & 0.25( \pm 0.01) \\ \text { Dark } & \text { Dark } & \text { NA } & \text { Blue } & 0.17( \pm 0.01) \\ \text { Dark } & \text { Light } & \text { NA } & \text { Red } & 0.30( \pm 0.02) \\ \text { Light } & \text { Dark } & \text { NA } & \text { Blue } & 0.30 \\ \text { Dark } & \text { Dark } & \text { Light } & \text { Red } & 0.08( \pm 0.02)\end{array}$

NA, Not applicable.

certain conditions of lighting (Fig. 1). The decrease in pyocyanin was dependent both on the intensity and on the wavelength of the light. Light with wavelengths in the ultraviolet and violet regions of the spectrum was primarily responsible for the decrease in pyocyanin concentration.

\section{Nature of light-mediated decrease in pyocyanin}

The effect of light on pyocyanin concentration could be due to a number of interactions including an indirect effect of light on the growth medium, a direct effect of light on the pyocyanin molecule or a direct effect of light on $P$. aeruginosa. When uninoculated growth medium was pre-irradiated with broad-spectrum light and subsequently inoculated with $P$. aeruginosa, cultures grown in the dark on unirradiated and pre-irradiated media had similar concentrations of pyocyanin (Table 1). Thus, the effect of light on pyocyanin was not due to an irreversible, indirect effect of light on the growth medium. Similar results were obtained with $371 \mathrm{~nm}$ light at $300 \mu \mathrm{W} \mathrm{cm}$ ch $^{-2}$ (data not presented). However, when plates with dark-grown $P$. aeruginosa had their micro-organisms removed and were subsequently exposed to broad-spectrum light (Table 1) or $371 \mathrm{~nm}$ light at $300 \mu \mathrm{W} \mathrm{cm}-2$ (data not presented), the pyocyanin concentration decreased. Thus it appears that light has a direct effect on pyocyanin after its release from bacteria.

\section{Photoinactivation of pyocyanin extracts}

To confirm that light affected pyocyanin directly, aqueous extracts of pyocyanin were exposed to broad-spectrum light and the photoinactivation of the pigment was observed as a function of time (Fig. 2). Photoinactivation of pyocyanin appeared to follow first-order kinetics. Pyocyanin had a half-life of about $36 \mathrm{~h}$. Incubation of the colourless photoproduct(s) in the dark for up to $4 \mathrm{~d}$ did not result in recoloration. Spectra of pyocyanin and its photoproduct(s) at various times (arrowed in Fig. 2) during the course of photoinactivation are shown in Fig. 3.

\section{DISCUSSION}

The results presented here show that exposure of $P$. aeruginosa cultures to light can produce changes in observable pigmentation. Pyorubrin and pyoverdin concentrations were either unaffected or only marginally affected by the lighting conditions tested, whereas the pyocyanin concentration decreased drastically. Subsequent studies indicated a direct 


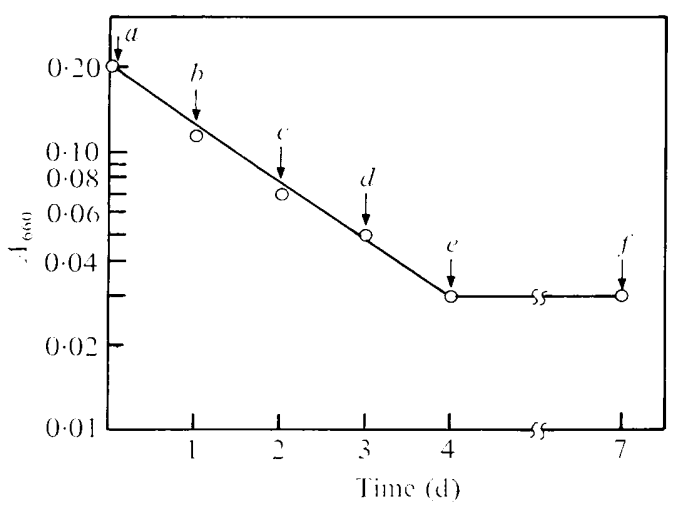

Fig. 2. Inactivation of pyocyanin extract by light. Pyocyanin, extracted as described in Methods, was thinly layered in Pyrex Petri dishes ( $6 \mathrm{ml}$ per $15 \times 60 \mathrm{~mm}$ dish) and exposed to broad-spectrum light at $2400 \mu \mathrm{W} \mathrm{cm}-2$. The Petri dishes transmitted $90 \%$ of the incident light above $345 \mathrm{~nm}$. At various times, samples were removed and their absorbance at $660 \mathrm{~nm}$ was determined.

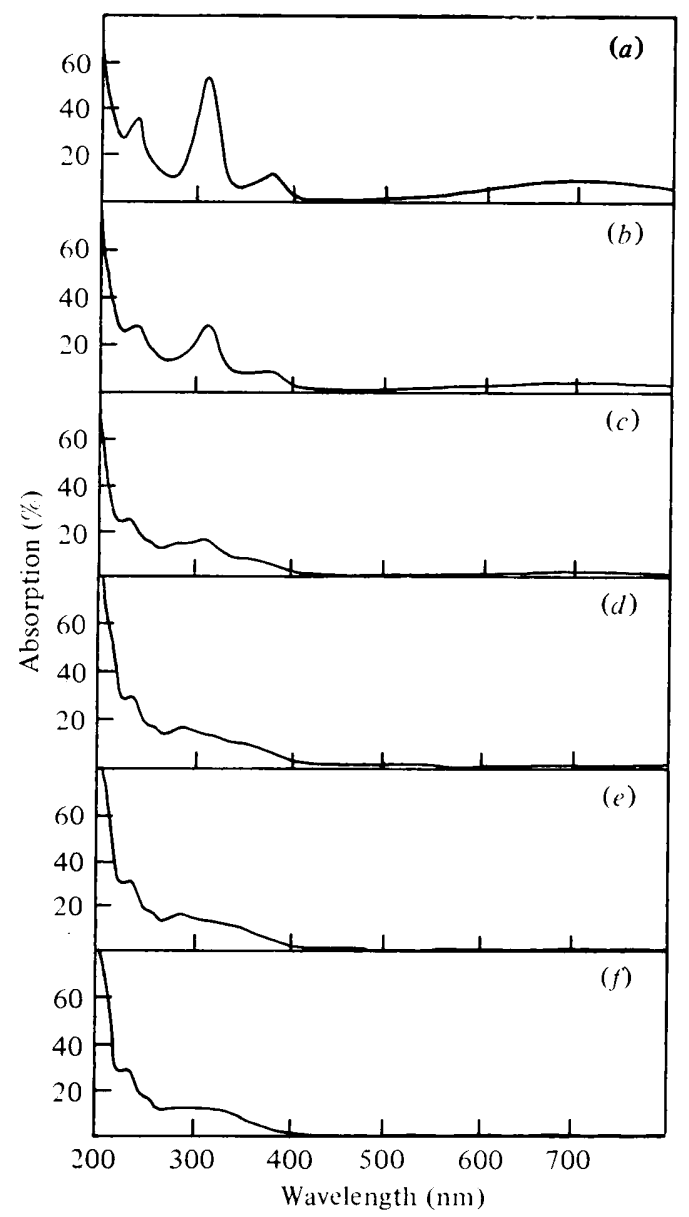

Fig. 3. Spectra of pyocyanin and its photoproduct(s). At various times during the photoinactivation of pyocyanin extracts (see Fig. $2 a$ to $f$ ), visible and ultraviolet spectra were recorded using a Perkin Elmer model 570 spectrophotometer. 
effect of light on the pigment, its inactivation resulting in the production of colourless photoproduct(s). The initial observations that dark-grown cultures produced bluepurple coloured plates while light-grown cultures produced red agar are thus explained by the fact that plates kept in the dark contain pyocyanin (blue) and pyorubrin (red) pigments whereas plates exposed to light contain pyorubrin (red) and the photoproduct of pyocyanin (colourless). Both light- and dark-grown cultures produce pyoverdin (yellow) which is too weak in colour to be visually detectable above the blue-purple and red backgrounds. The above findings also provide a likely explanation for the frequent observation in older literature that $P$. aeruginosa cultures show the gradual appearance of reddish brown colour when allowed to age in the laboratory exposed to 'air' (see Leonard, 1924).

Studies on pyocyanin both in situ (see Fig. 1) and in extracts (data not presented) showed that exposure to $371 \mathrm{~nm}$ (near ultraviolet) and $420 \mathrm{~nm}$ (violet) light resulted in the photoinactivation of this pigment, whereas $550 \mathrm{~nm}$ (green), $660 \mathrm{~nm}$ (red) and $740 \mathrm{~nm}$ (far red) light had little or no effect. Additional studies with pyocyanin extracts showed that this pigment is also strongly photoinactivated by light of wavelengths shorter than $371 \mathrm{~nm}$, specifically 254, 313 and $365 \mathrm{~nm}$ (data not presented). The shorter the wavelength of ultraviolet light, the more rapidly the inactivation occurred for any given intensity. At present, the photoproduct(s) of pyocyanin have not been isolated and the chemical structure(s) remain unknown. However, on the basis of the wavelengths of light absorbed, the nitrogen-containing tricyclic structure of pyocyanin, and the downward shift in ultraviolet spectra after irradiation (see Fig. 3), it seems most likely that the $\mathrm{N}$-containing ring is the site of disruption by light.

As noted above, pyocyanin is sensitive to photoinactivation by light of wavelengths shorter than $371 \mathrm{~nm}$. Elliott (1958) reported that pyoverdin extracts in neutral solution are also inactivated by 'short wavelength' ultraviolet light, with a resultant decrease in fluorescence. However, since no loss of fluorescence was observed in the present study, it appears that photoinactivation of pyoverdin does not occur in situ under the conditions tested.

The effect of light on pyocyanin is of interest for both scientific and clinical reasons. Although pyocyanin in situ in agar plates is reasonably well protected from photoinactivation by normal intensities of laboratory light (100 to $200 \mu \mathrm{W} \mathrm{cm}-2)$ and several days of continual exposure are needed in order to produce a visible colour change, extracted pigment is much more vulnerable. Care should be taken to prevent excessive exposure of pyocyanin extracts being used for quantitative or spectral studies to light, especially below $500 \mathrm{~nm}$. The use of pyocyanin production as a rapid identifying characteristic of $P$. aeruginosa is another good reason for continuing the usual practice of growing clinical cultures in the dark.

We would like to thank Dr Charles Botticelli and Mr Joseph Weidenheimer for critical evaluation of this manuscript, and Ms Barbara Schaller for expert technical help.

\section{REFERENCES}

ELLIOTT, R.P. (1958). Some properties of pyoverdine, the water-soluble fluorescent pigment of the pseudomonads. Applied Microbiology 6, 241-246.

JAGGER, J. (1972). Growth delay and photoprotection induced by near ultraviolet light. In Research Progress in Organic-Biological and Medical Chemistry, vol. III, pp. 383-401. Edited by U. Gallo \& L. Samtamiria. Amsterdam: North Holland.

KING, E. O., WARD, M. \& RANEY, D. (1954). Two simple media for the demonstration of pyocyanin and fluorescin. Journal of Laboratory and Clinical Medicine 44, 301-307.
LEACH, C. M. (1971). A practical guide to the effects of visible and ultraviolet light on fungi. Methods in Microbiology 4, 609-664.

LEONARD, V. (1924). Pyorubrin, a new pigment produced by Bacillus pyocyaneus. American Journal of Hygiene 4, 404-407.

Palumbo, S. A. (1972). Role of iron and sulfur in pigment and slime formation by Pseudomonas aeruginosa. Journal of Bacteriology 111, 430-436.

Propst-Ricciuti, C. \& KenNY, K. (1974). The effect of light on the growth of $\beta$-lactam antibiotic-producing fungi. Developments in Industrial Microbiology 17, 233-240. 
Siebert, M., Wetherbee, P. J. \& Job, D. D. (1975). The effects of light intensity and spectral quality on growth and shoot initiation in tobacco callus. Plant Physiology 56, 130-139.

TAYlOR, B. L. \& Koshland, D. E., JR (1975). Intrinsic and extrinsic light responses of Salmonella typhimurium and Escherichia coli. Journal of Bacteriology 123, 557-569.
Tномаs, G. (1977). Effects of near-ultraviolet light on microorganisms. Photochemistry and Photobiology 26, 669-673.

Zelle, M. R. \& Hollaender, A. (1955). Effects of radiation on bacteria. In Radiation Biology. Edited by A. Hollaender. New York: McGraw-Hill. 